\title{
Does one bout of high intensity resistance training change circulatory levels of irisin?
}

Maria Fernandez det-Valle PhD, Matthew Short MS', Jacalyn J. McComb PhD FACSM1', Eunhee Chung PhD', Shellby Kloiber MS', Eneko Larumbe-Zabala PhD² ${ }^{1}$ Department of Kinesiology and Sport Management, Texas Tech University, Lubbock, TX.
${ }^{2}$ Clinical Reserch Instinute. Texas Tech University HSC, Lubbock, TX.

\begin{tabular}{l} 
Introduction \\
The recently novel identified myokine, irisin, has \\
gained attention as a way to increase energy \\
expenditure by enhancing metabolic function. \\
Exercise and active lifestyle increase the synthesi s of \\
contraction-regulated myokines that have direct effect \\
on cells metabolism. \\
The objective of this study is to analyze the effects of \\
one bout of high intensity exercise on circul atory \\
levels of irisin in healthy young adults. \\
\hline Methods \\
- Sample: \\
A total of 24 participants were recruited. Subjects \\
were blocked by sex, BMI, and LBM and randomized \\
to either control (n-13) or intervention (n-11) (Table \\
1). One control was excluded for not fulfill ing the \\
inclusion criteria (Figure 1). \\
- Assessments: \\
Physical fitness was assessed through Body \\
Composition, Muscular Strengh, Cardiorespiratory \\
Fitness, and irisin Levels. Strength assessments were \\
performed after 3 familiarization sessions in order to \\
acquire appropriate exercise techniques, and prevent \\
the learning effect (Figure 1). \\
- Intervention: \\
High-Intensity circuit training consisting on 7 whole \\
body exercises, $3 x 10$ with resting periods of 30 \\
seconds/exercises and 1 -2 min/sets Intensity was set \\
at $7-10$ of the 10 -point Rating of Perception Exertion \\
(RPE) scale [1]. Lactate levels were measured to \\
cross-check intensity (Figure 2).
\end{tabular}

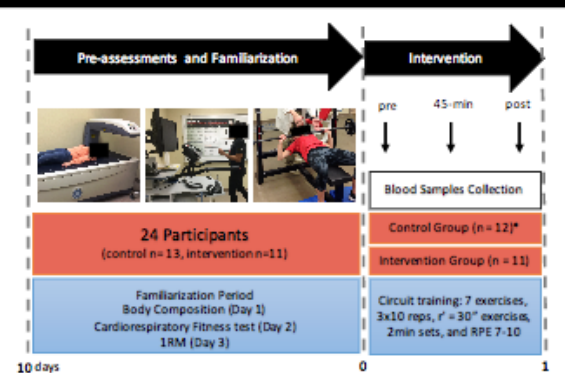

10dars Figure 1. Pre-æs ses sment, famllarizaion, and intervertion summary

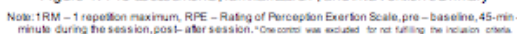

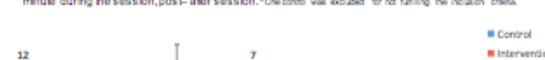

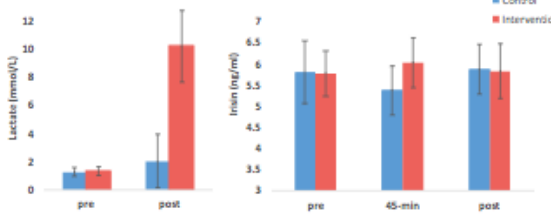

Figure 2. Lactze levels Figure 3 . Insin responses to acte exercise

\section{Results}

Interaction effect (time $\mathrm{x}$ intervention) $(\mathrm{F}[2,42]-2.281, \mathrm{p}=0.115)$ time $(\mathrm{F}[2,42]=0.325, \mathrm{p}=0.724)$ and intervention $(\mathrm{F}[1,21]=0.239$, $\mathrm{p}=0.630$ ) effects were not significant (Figure 3 ) when circulating irisin levels $(\mathrm{ng} / \mathrm{mL})$ were analyzed.
Table 1. Sample descriptive

\begin{tabular}{|c|c|c|c|c|}
\hline & \multicolumn{2}{|c|}{$\begin{array}{c}\text { Control } \\
(\mathrm{n}-12)\end{array}$} & \multicolumn{2}{|c|}{$\begin{array}{c}\text { Intervention } \\
(\mathrm{n}-11)\end{array}$} \\
\hline & Mean & SD & Mean & SD \\
\hline Weight $(\mathrm{kg})$ & 69.01 & 10.73 & 63.74 & 7.06 \\
\hline Height $(\mathrm{cm})$ & 173.02 & 0.09 & 170.83 & 0.09 \\
\hline BMI $\left(\mathrm{kg} / \mathrm{m}^{2}\right)$ & 22.92 & 2.13 & 21.81 & 1.35 \\
\hline LBM (kg) & 49.18 & 11.58 & 47.32 & 10.95 \\
\hline lñsin $(\mathrm{ng} / \mathrm{m} \mid)^{*}$ & 5.80 & 1.31 & 5.77 & 0.91 \\
\hline
\end{tabular}

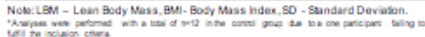

\section{Discussion and Conclusion}

In addition to heterogeneous research findings, the lack of changes on serum concentrations of irisin after a resistance training intervention shown in this study adds controversial resuls to the literature [2 4].

If irisin is an exercise-induced hormone, other confounding variables such as room temperature, body temperature, daily variation, physical fitness levels, or exercise intensity, and more rigorous designs - with control group - might be critical factors for future studies.

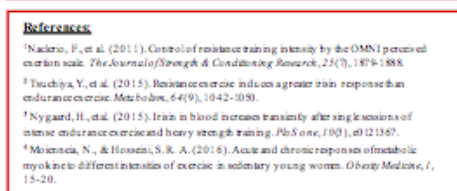

Acknowledgements: CALUE, FY2015 Scholarship Catalyst Program at TTU, participants, and Research Assistants. 